\title{
Traue einer Statistik, die du nicht selbst erstellt hast
}

\author{
Katrin Schmidt • Thomas Weißbrodt
}

Eingegangen: 22. Februar 2019 / Angenommen: 26. Juni 2019 / Online publiziert: 15. Juli 2019

(C) Der/die Autor(en) 2019

Zusammenfassung Wir alle benötigen ein grundlegendes Verständnis von Statistik, um mit der wachsenden Menge an verfügbaren statistischen Daten, Analysen und Ergebnissen, die uns täglich begegnen, umgehen zu können. Auf dieser Basis können Entscheidungen fundiert getroffen und Fehlschlüsse reduziert werden. Der Arbeitsmarkt gehört zu den Themenfeldern, die für viele Menschen wichtig und in ihrem Alltag präsent sind. Die Daten dazu sind umfänglich und die Konzepte mitunter komplex. Die Statistik der Bundesagentur für Arbeit unterbreitet ihren Nutzern daher verschiedene Angebote, um das Verständnis für Statistik generell, aber natürlich speziell für die Daten und Zusammenhänge hinsichtlich des Arbeitsmarkts zu verbessern. Im nachfolgenden wird zunächst die Aufgabe der Statistik der Bundesagentur für Arbeit als Teil des Systems amtlicher Statistik dargestellt. Es geht auch darum zu erfahren, welche Aufgaben die Statistik der Bundesagentur für Arbeit hat. Im Anschluss werden zwei häufig auftretende Missverständnisse bezüglich zentraler Arbeitsmarktfragen erläutert: Die Zusammenhänge von Arbeitslosigkeit, Verfügbarkeit am Arbeitsmarkt, Arbeitsuche und den Bezug von Leistungen der Grundsicherung. Bei bestimmten Personengruppen und Status zeigen sich Überschneidungen, andere schließen sich dagegen aus - bekannt ist das in der Öffentlichkeit nur zum Teil. Deshalb wird auch dargestellt, welche Ansätze die Statistik der Bundesagentur für Arbeit verfolgt, um einem möglichst breiten Kreis von Interessenten jeweils adressatengerecht Daten, Kontextinformationen und Analysen zur Verfügung zu stellen und die Nutzer bei der Interpretation von Arbeitsmarktdaten zu unterstützen.

\footnotetext{
K. Schmidt

Statistik der Bundesagentur für Arbeit, Nürnberg, Deutschland

E-Mail: katrin.schmidt8@arbeitsagentur.de

T. Weißbrodt $(\bowtie)$

Statistik der Bundesagentur für Arbeit, Frankfurt a. M., Deutschland

E-Mail: thomas.weissbrodt@arbeitsagentur.de
} 
Schlüsselwörter Statistical Literacy · Amtliche Statistik · Arbeitsmarkt ·

Arbeitslosigkeit · Unterbeschäftigung · Grundsicherung für Arbeitsuchende

JEL E24 $\cdot \mathrm{I} 38 \cdot \mathbf{J} 21 \cdot \mathrm{J} 64$

\section{Trust a statistic that you didn't create yourself}

Abstract We all need a basic understanding of statistics to be able to handle the increasing amount of statistical data, analyses and results that we encounter every day. On this basis, we can make informed decisions and reduce the number of mistakes. The labor market is one of the topics that are important for many people and present in their everyday lives. The amount of data is extensive and the concepts can sometimes be complex. The Federal Employment Agency's statistics division therefore offers their users various ways to improve their understanding of statistics in general, but of course especially for data and contexts regarding the labor market. In the following, the function of the Federal Employment Agency's statistics division within the whole system of official statistics is explained. It is also describes the tasks of the Federal Employment Agency's statistics division. Furthermore, the article explains two common misunderstandings about the central labor market: the link between unemployment, availability on the labor market and job search as well as the entitlement to basic benefits ("Grundsicherung"). For certain groups of people and status there are overlaps, while others are mutually exclusive-a phenomenon little of which is known to the public. Therefore this article also describes the approach the Federal Employment Agency's statistics division is pursuing in order to make data, context information and analyses available to as many people as possible and to assist users in the interpretation of labor market data.

\section{Einleitung}

Die Nutzung von Statistiken ist nichts Neues, aber nie zuvor hatten wir alle täglich mit so vielen statistischen Daten, Analysen und Ergebnissen zu tun. Digitale Fortschritte erweitern die statistischen Möglichkeiten, die wiederum zu weiter differenzierten Datenanfragen führen - und umgekehrt. Diese Entwicklungen lassen sich auch bei der Statistik der Bundesagentur für Arbeit (BA) (das ist die Bezeichnung der Statistikabteilung der BA) nachvollziehen. Heute werden Arbeitsmarktstatistiken aus verschiedenen Quellen verknüpft. Bestimmte Personengruppen können eindeutiger voneinander abgegrenzt werden. Laufend werden Messkonzepte und Berichtssysteme verbessert. Bei Datenausfällen helfen Imputationsverfahren fehlende Werte zu schätzen. In den Daten werden mit Saisonbereinigungsverfahren jahreszeitliche Einflüsse eliminiert. Es werden regelmäßig Antworten auf aktuelle politische Fragen gegeben, darunter werden auch ad hoc Näherungslösungen entwickelt und verschiedene Blickwinkel zur Beleuchtung eines Themas eingenommen. Das Datenangebot ist stark gestiegen - und dieses Angebot ist erforderlich, um Zugänge zu den komplexen Arbeitsmarktthemen zu finden. 
Die Statistik der BA wird in verschiedenen Kontexten von sehr vielen Menschen wahrgenommen. Die Zahlen haben eine zentrale Bedeutung, weil über sie als Indikatoren für Entwicklungen auf dem Arbeitsmarkt in den Medien berichtet wird. In diesem Zusammenhang werden der Statistik der BA in der Öffentlichkeit verschiedentlich Tricksereien, mangelnde Objektivität und Neutralität nachgesagt. Es wird immer wieder der Vorwurf laut, die Zahlen seien schöngefärbt und würden beispielsweise nicht die wahre Höhe der Arbeitslosigkeit abbilden. Diese Debatten lassen sich heute am einfachsten in den Kommentarspalten aller Onlineveröffentlichungen zum Thema nachverfolgen. Warum ist das so? Und was unternimmt die Statistik der BA selbst dagegen? Unbeachtet bleibt oft, dass es nicht die alles erklärende eine Zahl gibt, sondern ein stark differenziertes Geflecht verschiedener Indikatoren, basierend auf gesetzlichen Vorgaben.

Nachfolgend werden zunächst anhand von Beispielen diese Sachverhalte dargestellt. Danach wird gezeigt, was die Statistik der BA unternimmt, um einem möglichst breiten Kreis von Interessenten adressatengerecht Daten, Kontextinformationen und Analysen zur Verfügung zu stellen und die Nutzer bei der Interpretation zu unterstützen.

\section{Was macht die Statistik der Bundesagentur für Arbeit und welche Aufgaben hat sie?}

Die Statistik der BA stellt Bürgerinnen und Bürgern, Medien, Wirtschaft, Politik, Wissenschaft und Verwaltung Informationen über den Arbeitsmarkt bereit: Sie veröffentlicht regelmäßig Daten zu Arbeitslosigkeit und Unterbeschäftigung, gemeldeten Stellen, sozialversicherungspflichtiger und geringfügiger Beschäftigung, arbeitsmarktpolitischen Maßnahmen, zur Grundsicherung für Arbeitsuchende, Geldleistungen und zum Ausbildungsmarkt. Sie führt Analysen durch, erläutert statistische Zusammenhänge und Hintergründe, erteilt Auskünfte, berät und erstellt Sonderauswertungen. Ziel ist es, den am Arbeitsmarkt agierenden Institutionen sichere Grundlagen zur Einschätzung der Gesamtsituation, von Teilarbeitsmärkten und der regionalen Entwicklungen zur Verfügung zu stellen. Handlungsbedarfe können so rechtzeitig erkannt und entsprechend gesteuert werden.

Die Statistik der BA erstellt ihre Statistiken auf Grundlage verschiedener Bücher des Sozialgesetzbuches (SGB): $§ 281$ SGB III regelt die Statistiken über Arbeitslosigkeit, Beschäftigung, Arbeitsförderung und Migrationshintergrund, § 53 SGB II die Statistiken über die Grundsicherung für Arbeitsuchende. Die Statistik der BA nimmt innerhalb der Bundesagentur für Arbeit insofern eine Sonderrolle ein, als nach $\S 283$ Satz 1 SGB III die Arbeitsmarktstatistiken dem die Fachaufsicht ausübenden Bundesministerium für Arbeit und Soziales vorzulegen und in geeigneter Form zu veröffentlichen sind. Sie arbeitet mit anderen Institutionen wie dem Statistischen Bundesamt, den Statistischen Landesämtern, Statistischen Stellen in den Kommunen, dem Institut für Arbeitsmarkt und Berufsforschung der BA, dem Bundesinstitut für Berufsbildungsforschung und Verbänden, z.B. dem Verband der Deutschen Städtestatistiker und der Deutschen Statistischen Gesellschaft zusammen. So finden Leitungs- und Fachtreffen mit nationalen und europäischen Institutionen amtlicher 
Statistik ebenso statt wie themenspezifische Kooperationen etwa bei der Überarbeitung der Klassifizierung der Berufe (KldB 2010) oder bei einem gemeinsamen Datenangebot von Statistik der BA, Statistischem Bundesamt und dem Bundesamt für Migration und Flüchtlinge.

Aber woher kommen die Daten eigentlich? Die Statistikdaten zum Arbeits- und Ausbildungsmarkt, der Grundsicherung, der Maßnahmen der aktiven Arbeitsförderung sowie der Entgeltersatzleistungen basieren auf Geschäftsdaten, die bei der Bundesagentur für Arbeit und den Jobcentern im Rahmen der Aufgabenerfüllung nach dem SGB II und SGB III anfallen. Es handelt sich dabei stets um Vollerhebungen, so dass die Daten auch in tiefer regionaler Gliederung fachlich umfassend differenziert dargestellt werden können. Diese Prozessdaten werden für die statistische Verwendung ,übersetzt“. Für die operativen Daten werden Konzepte und Methoden geschaffen, um sie überhaupt statistisch nutzen zu können. Für die Berichterstattung zum Thema Beschäftigung werden die Daten durch Arbeitgebermeldungen zu sozialversicherungspflichtig und geringfügig Beschäftigten gewonnen.

$\mathrm{Zu}$ dem Spektrum der statistischen Aufgaben zählen die Definition der statistischen Größen und Inhalte, die Organisation des Datenerhebungsprozesses, die Festlegung der Zähl- und Auswertungsmethoden, die Entwicklung von Hochrechnungsund Schätzverfahren bei Datenausfällen und bei vorläufigen Daten mit Wartezeit sowie die Qualitätssicherung der Ausgangsdaten und der statistischen Ergebnisse. Die Daten werden in Qualitäts- und Methodenberichten beschrieben, es werden statistische Berichtssysteme entwickelt und das Internetangebot gepflegt. Auch der richtige Umgang mit Fehlern in Veröffentlichungen gehört hier dazu: Die Statistik der BA korrigiert die Fehler und informiert und benennt über Newsletter und Homepage die Korrekturen entsprechend.

Die Verpflichtung zur Objektivität, Neutralität und statistischen Geheimhaltung sowie zur wahrheitsgetreuen Ermittlung, Aufbereitung, Darstellung und Veröffentlichung der Daten sind die grundlegenden Prinzipien der amtlichen Statistik, wie sie sich aus dem Bundesstatistikgesetz und dem Verhaltenskodex für Europäische Statistiken (2017) ergeben. Dieser Kodex gibt ein ganzes Bündel an Standards für die Entwicklung, die Erstellung und Verbreitung von europäischen Statistiken verbindlich vor. Der Kodex selbst wird immer wieder überarbeitet um neueren Entwicklungen wie technologischen Fortschritten oder Änderungen der rechtlichen Rahmenbedingungen Rechnung zu tragen.

Die Einhaltung des Verhaltenskodex im Europäischen Statistischen System (ESS) wird durch Eurostat überwacht. Einen wichtigen Bestandteil der Strategie des ESS für die Umsetzung des Verhaltenskodex bilden Selbstbewertungen und gegenseitige Bewertungen der statistischen Ämter (Peer Reviews). Die Statistik der BA ist durch das Statistische Bundesamt in die Peer Reviews einbezogen.

Über diese institutionellen Vorgaben hinaus bietet die Statistik der BA verschiedene Möglichkeiten zur Unterstützung im Umgang mit unseren Arbeitsmarktstatistiken an, auf die in Abschn. 4 näher eingegangen wird. 


\section{Vorwurf: Die Statistik der BA rechnet schön}

Die deutsche Wirtschaft erlebte im Jahr 2017 einen kräftigen Aufschwung. Der Konjunkturaufschwung war breit angelegt und wurde vor allem von binnenwirtschaftlichen Kräften getragen. Auch der Arbeitsmarkt und speziell die Arbeitslosigkeit entwickelten sich sehr gut. Doch kann man dafür die Daten der BA überhaupt heranziehen? Denn trotz aller institutioneller Vorgaben wird der Statistik angekreidet, sie bilde nicht das ab, was Arbeitslosigkeit sei. Oder: Die Statistik der BA rechne Arbeitslose aus der Statistik heraus und lasse sie verschwinden. Der Grund für das Misstrauen in die Statistik ist einfach erklärbar: Das Alltagsverständnis von dem, was sich hinter dem Begriff der Arbeitslosigkeit verbirgt, ist abgekoppelt davon, wie Arbeitslosigkeit nach dem Sozialgesetzbuch definiert ist. Zunächst einmal ist die Bedeutung von Arbeitslosigkeit im Alltagsverständnis keineswegs falsch. Häufig verstehen Menschen unter Arbeitslosigkeit das Fehlen von Arbeit. Oder nichtvorhandene bezahlte Arbeit. Manchmal kommt noch die Annahme hinzu, dass keine Arbeit vorhanden ist, obwohl Arbeit gesucht wird. Komplexer wird die Annäherung, wenn man von Arbeitslosigkeit spricht, sofern sie unfreiwillig ist und die betroffenen Personen entsprechend bereit sind, Arbeit aufzunehmen bzw. ihre Arbeitskraft anzubieten.

Die vielseitigen Informationsangebote der Statistik der BA und die damit vorhandene Transparenz zeigen die Arbeiten daran, Begriffe wie Arbeitslosigkeit, Arbeitslosengeld als Versicherungsleistung, Arbeitslosengeld II - auch „Hartz IV“ genannt - als Leistung der Grundsicherung, in ein statistisches Alltagsverständnis zu integrieren. Die klaren begrifflichen und statistischen Abgrenzungen verschiedener Konstruktionen sind komplex, aber in dieser Form notwendig, weil dahinter immer ganz spezifische Personengruppen stehen, die mehr oder weniger weit vom Arbeitsmarkt entfernt sind. Jede dieser inhaltlichen Konstruktionen und ihre statistischen Abbildungen sind berechtigt, weil sich der Arbeitsmarkt und die vielen dazu gehörenden Fragen nicht mit einer Zahl abbilden und beschreiben lassen. Gleichzeitig bestehen für die Merkmalsträger, seien es Arbeitslose, Leistungsbezieher, Langzeitleistungsbezieher, Langzeitarbeitslose, Nichtarbeitslose Leistungsbezieher, Nichtarbeitslose Arbeitsuchende usw. mehr oder weniger große Schnittmengen, die meist über das geschilderte Alltagsverständnis hinausreichen. Anhand zweier Beispiele wird gezeigt, dass die Statistik der BA nicht schönfärbt, sondern sich um größtmögliche Transparenz bemüht.

\subsection{Wer in der Statistik als arbeitslos zählt und wer nicht}

Die Statistiken über arbeitslose Menschen ${ }^{1}$ informieren über Umfang und Struktur der Arbeitslosigkeit (vgl. Bundesagentur 2019a). Das Missverständnis bei dieser Zahl lautet, die Statistik der BA veröffentliche nicht die wahre Arbeitslosenzahl, sondern rechne etliche Arbeitslose heraus, um Erfolge für sich, die Bundesagentur für Arbeit oder die Regierung verbuchen zu können. Dieser Vorwurf trifft nicht zu,

\footnotetext{
1 Weiterführende Informationen zu den Inhalten der nachfolgenden Kapitel: Siehe Literaturverzeichnis.
} 
weil die Statistik der BA Arbeitslosigkeit so abbildet, wie sie im Sozialgesetzbuch (SGB) definiert ist.

In § 16 Absatz 1 SGB III wird Arbeitslosigkeit festgelegt: Arbeitslose sind Personen, die

- vorübergehend nicht in einem Beschäftigungsverhältnis stehen

- eine versicherungspflichtige Beschäftigung suchen

- den Vermittlungsbemühungen der Agentur für Arbeit zur Verfügung stehen

- sich bei der Agentur für Arbeit arbeitslos gemeldet haben.

Nach dieser Abgrenzung gelten also längst nicht alle erwachsenen Menschen, die keiner Erwerbsarbeit nachgehen, als arbeitslos. So nimmt die Arbeitslosenstatistik nach dem Sozialgesetzbuch eine sozialpolitische Perspektive ein: Es werden z.B. Schüler und Studenten, Frauen und Männer, die sich der Erziehung ihrer Kinder widmen, sowie Rentner und Pensionäre nicht als Arbeitslose angesehen. Auf der anderen Seite werden Personen als arbeitslos erfasst, die eine nur geringfügige Beschäftigung mit weniger als 15 Wochenstunden ausüben - sofern sie auf der Suche nach einer sozialversicherungspflichtigen Beschäftigung sind.

Allerdings wird die gesetzlich festgelegte Zählweise immer wieder etwas angepasst. So wurden durch die so genannten Hartz-Reformen im Jahre 2005 Hunderttausende erwerbsfähige Sozialhilfeempfänger, die weit vom Arbeitslosenstatus entfernt waren, in der Arbeitslosenstatistik sichtbar - die bis dahin existierende Arbeitslosen- und Sozialhilfe für Erwerbsfähige wurde durch die Grundsicherung für Arbeitsuchende abgelöst. Aber auch Veränderungen in die andere Richtung werden immer wieder durchgeführt, so dass die Arbeitslosenzahl reduziert wird. Es heißt in $\S 16$ Absatz 2 SGB III: „Teilnehmer an Maßnahmen der aktiven Arbeitsmarktpolitik gelten als nicht arbeitslos“. Hierzu zählen alle Teilnahmen an Maßnahmen zur Aktivierung und beruflichen Eingliederung nach § 45 SGB III. Diese Festlegung stellt den ersten eigentlichen Kritikpunkt an der Statistik dar. Schließlich wären diese Personen ohne Teilnahme an einer dieser Maßnahmen sehr wahrscheinlich arbeitslos. Um diese Kritik abzumildern, veröffentlicht die Statistik der BA monatlich zu den Arbeitslosenzahlen die Statistik zur Unterbeschäftigung (vgl. Bundesagentur für Arbeit 2009 und 2013). Diese macht transparent, wie viele Menschen ihren Wunsch nach einer Beschäftigung nicht realisieren können, indem zusätzlich zu den Arbeitslosen die Personengruppen abgebildet werden, die sich aufgrund einer Maßnahmeteilnahme oder eines anderen Sonderstatus sehr nahe bis etwas weiter entfernt vom Arbeitslosenstatus nach $\S 16$ Abs. 1 SGB III befinden. Sie reduzieren die Arbeitslosenzahl, weil die Teilnahme an einer Förderung oder die Verweilzeit in einem Sonderstatus das tatsächliche Arbeitskräfteangebot verringern und gesamtwirtschaftlich entlastend wirken.

Dies gilt zuallererst für Maßnahmen zur Aktivierung und beruflichen Eingliederung und vorruhestandsähnliche Regelungen. Zu letzterem ist für Arbeitslosengeld II-Bezieher nach § 53a SGB II die Ausnahme definiert, dass sie nach Vollendung des 58. Lebensjahres und einem Leistungsbezug in der Grundsicherung für eine Dauer von mindestens zwölf Monaten dann nicht als arbeitslos gelten, wenn ihnen in diesem Zeitraum keine sozialversicherungspflichtige Beschäftigung angeboten worden ist. Diese Regelung ist der zweite bedeutsame Kritikpunkt an der 
plus Personen, weit weg vom Arbeitslosenstatus (Unterbeschäftigung)

plus Personen, nahe am Arbeitslosenstatus (Unterbeschäftigung im engeren Sinne)

plus Personen, im weiteren Sinne arbeitslos

Arbeitslose

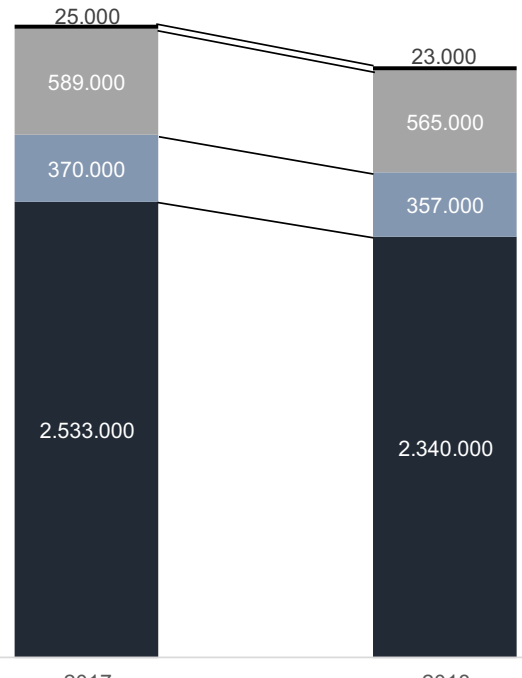

2017

2018

Abb. 1 Unterbeschäftigung, Deutschland, 2017 und 2018

Statistik - zunehmend auch vor dem Hintergrund eines sich nach hinten verschiebenden Renteneintrittsalters. In der statistischen Darstellung werden diese beiden Sachverhalte aufgrund der unmittelbaren Nähe zur Arbeitslosigkeit als Arbeitslosigkeit im weiteren Sinne bezeichnet.

Bei Personen in verschiedenen Qualifizierungsmaßnahmen, Beschäftigten am 2. Arbeitsmarkt (darunter vor allem in sogenannten Arbeitsgelegenheiten (AGH)) sowie bei kurzfristiger Arbeitsunfähigkeit besteht ebenfalls eine Nähe zum Arbeitslosenstatus. Im Konzept werden sie als Unterbeschäftigung im engeren Sinne bezeichnet.

Weiter entfernt vom Arbeitslosenstatus, aber in Maßnahmen, die gesamtwirtschaftlich entlasten, sind Personen mit einem Gründungzuschuss zur Förderung der Selbständigkeit und Kurzarbeiter. Mit diesen Gruppen wird das Konzept als Unterbeschäftigung vervollständigt.

Abb. 1 stellt den Vorteil dieses Konzeptes dar, das umfassender als die Arbeitslosigkeit abbildet, wie viele Menschen ihren Wunsch nach einer Beschäftigung nicht realisieren können: Die Unterbeschäftigung ist robust gegenüber dem Einsatz von entlastenden arbeitsmarktpolitischen Maßnahmen. Diese Statistik ermöglicht bessere Vergleiche, indem ein unterschiedlich stark ausgeprägter Einsatz von arbeitsmarktpolitischen Maßnahmen die Zahlen räumlich wie zeitlich nicht beeinflusst. Sie ist rechtskreisübergreifend und jahresdurchschnittlich von 2017 auf 2018 um 231.000 bzw. 6,6\% auf 3,286 Mio. zurückgegangen. Im Vergleich dazu sank die Arbeitslosigkeit im selben Zeitraum um 193.000 bzw. 7,6\%. Die Unterbeschäftigung als Form einer transparenten Darstellung wird monatlich in tiefer regionaler Gliederung veröffentlicht. 


\subsection{Sind alle Bezieher von Arbeitslosengeld II arbeitslos?}

Die Berichterstattung zur Grundsicherung für Arbeitsuchende (SGB II) erfolgt ebenfalls monatlich und regional tief gegliedert. Die statistischen Informationen über die Gewährung von Leistungen zum Lebensunterhalt werden aus Geschäftsdaten generiert. Das Beantragungsverfahren ist insgesamt komplexer als das zu Leistungen aus der Arbeitslosenversicherung. Um nachträgliche Bewilligungen und Aufhebungen in der Statistik berïcksichtigen zu können, beträgt die Wartezeit bis zur Veröffentlichung festgeschriebener Daten drei Monate. Leistungen aus der Grundsicherung für Arbeitsuchende erhalten Personen, die den gemeinsamen Lebensunterhalt mit den gemeinsam im Haushalt lebenden Personen nicht oder nicht vollständig bestreiten können, die also hilfebedürftig sind. Die Aufgabe der Grundsicherungsstatistik ist es, über die Personen und Bedarfsgemeinschaften zu berichten, die Leistungen nach dem SGB II erhalten, darunter vor allem Arbeitslosengeld II für erwerbsfähige Leistungsberechtigte und Sozialgeld für nicht erwerbsfähige Leistungsberechtigte (vgl. Bundesagentur für Arbeit 2018).

An dieser Stelle setzt unmittelbar das häufige Missverständnis in Bezug auf die Empfänger von Arbeitslosengeld II an: Bei diesen Personen, landläufig auch als „Hartz IV-Bezieher" bezeichnet, handele es sich um Langzeitarbeitslose, also um Personen, die seit einem Jahr oder länger arbeitslos gemeldet sind. Das stimmt nur zum Teil. Richtig ist: Diese Statistik bildet Personen in sehr heterogenen Lebenslagen ab, die längst nicht alle den Status der Arbeitslosigkeit haben (vgl. Bundesagentur für Arbeit 2015). Die beiden in der Berichterstattung zentralen Personengruppen in Bedarfsgemeinschaften werden nachfolgend beschrieben: Erwerbsfähige Leistungsberechtigte (ELB) und nicht erwerbsfähige Leistungsberechtigte bilden gemeinsam die Gruppe der Regelleistungsberechtigten. Darüber hinaus sind noch weitere Differenzierungen von Personengruppen möglich, auf die an dieser Stelle nicht weiter eingegangen wird.

Als erwerbsfähige Leistungsberechtigte mit Anspruch auf Arbeitslosengeld II gelten gemäß § 7 SGB II Personen, die

- das 15. Lebensjahr vollendet und die Altersgrenze nach § 7a SGB II (Renteneintrittsalter) noch nicht erreicht haben

- erwerbsfähig sind, d.h. in der Lage, täglich für mindestens drei Stunden zu arbeiten

- hilfebedürftig sind

- ihren gewöhnlichen Aufenthalt in der Bundesrepublik Deutschland haben.

Nicht erwerbsfähige Leistungsberechtigte mit Anspruch auf Sozialgeld sind in der Regel meist die Kinder unter 15 Jahren, die in einer Bedarfsgemeinschaft mit ihren erwerbsfähigen Eltern leben - bundesweit etwa 1.700.000. Allgemein sind nach der Definition aber auch Personen ab 15 Jahren gemeint, die z.B. aufgrund gesundheitlicher Einschränkungen nicht den Kriterien der Erwerbsfähigkeit entsprechen quantitativ handelt es sich dabei aber um sehr wenige Personen.

Das Arbeitslosengeld II - oder eben Hartz IV - ist nicht an den Status der Arbeitslosigkeit geknüpft (vgl. § 7 SGB II i. V. mit § 53a SGB II). In Abb. 2 werden anhand proportionaler Flächen die realen Verhältnisse einzelner Größen zueinander 


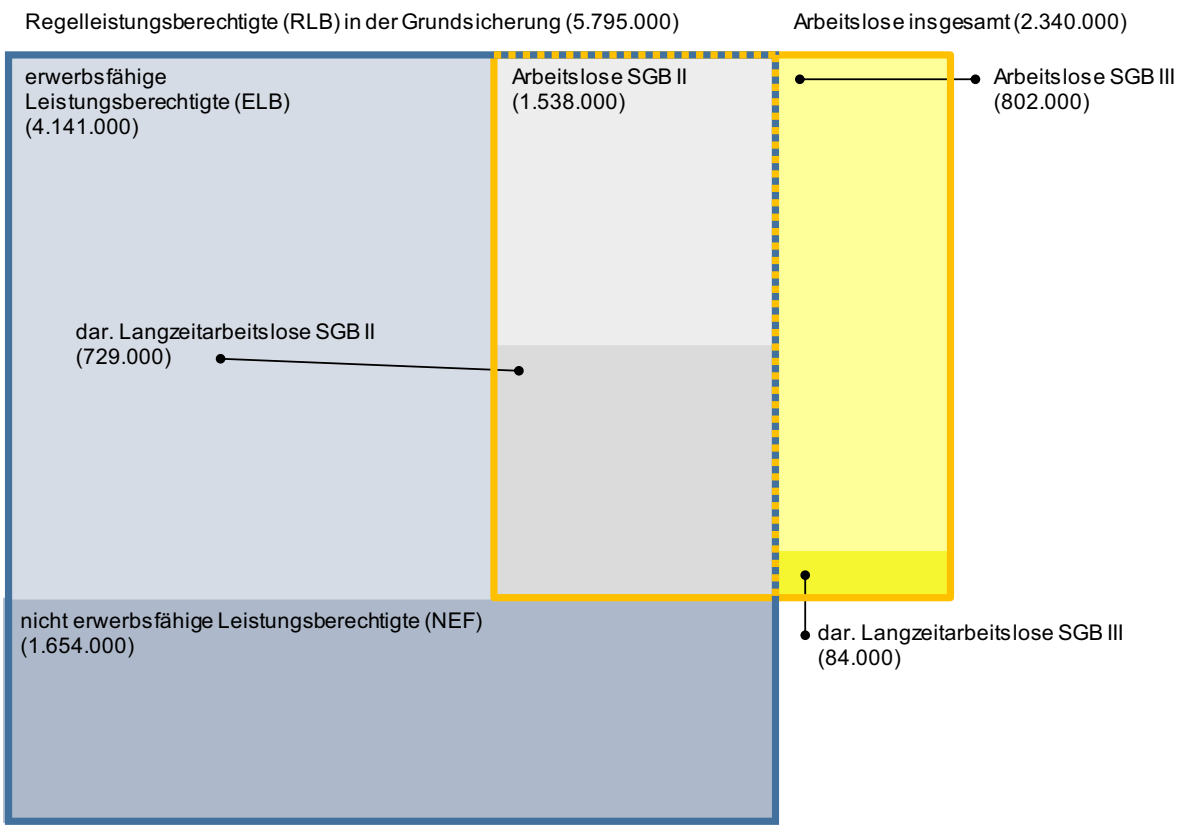

Abb. 2 Regelleistungsberechtigte in der Grundsicherung und Arbeitslose nach Rechtskreisen, Deutschland, 2018

gezeigt. Diese Abgrenzungen machen deutlich sichtbar, dass es sich bei den mehr als 4.100.000 erwerbsfähigen Leistungsberechtigten im SGB II nur zu einem kleineren Teil um Arbeitslose (1.500.000) und darunter um Langzeitarbeitslose (700.000) handelt. Gleichwohl ist die Zahl Langzeitarbeitsloser in der Grundsicherung nach dem SGB II erheblich höher als in der Arbeitslosenversicherung nach dem SGB III. Dies lässt sich damit erklären, dass Arbeitslose, die ein Jahr und länger arbeitslos sind, in der Regel in die Anspruchsgrundlage des SGB II wechseln².

Langzeitarbeitslosigkeit oder Arbeitslosigkeit insgesamt sind somit nicht die dominierenden Merkmale in der Grundsicherung. Arbeitslos sind unter den erwerbsfähigen Leistungsberechtigten im SGB II etwas mehr als ein Drittel. Die Bedingungen, nach denen erwerbsfähige Leistungsberechtigte den Status der Arbeitslosigkeit aufweisen, entsprechen den beschriebenen Kriterien im vorigen Kapitel: Beschäftigungslosigkeit, Suche, Verfügbarkeit, Meldung. Es gibt typische Konstellationen im SGB II, in denen Arbeitslosengeld II-Bezieher nicht als arbeitslos geführt werden: Zum Beispiel werden beschäftigte Personen, die mindestens $15 \mathrm{~h}$ in der Woche arbeiten, aber wegen zu geringen Einkommens bedürftig nach dem SGB II sind und deshalb Arbeitslosengeld II erhalten, nicht als arbeitslos gezählt, weil das Kriterium der Beschäftigungslosigkeit nicht erfüllt ist. Erwerbsfähige Leistungsberechtigte,

\footnotetext{
2 Langzeitarbeitslose im SGB III haben entweder keinen Anspruch auf Leistungen der Arbeitslosenversicherung - etwa Berufseinsteiger, Berufsrückkehrer oder Langzeitarbeitslose, die wegen fehlender Bedürftigkeit kein Arbeitslosengeld II erhalten. Außerdem sind hier ältere Arbeitslosengeldempfänger enthalten, die Leistungsansprüche von mehr als zwölf Monaten haben.
} 


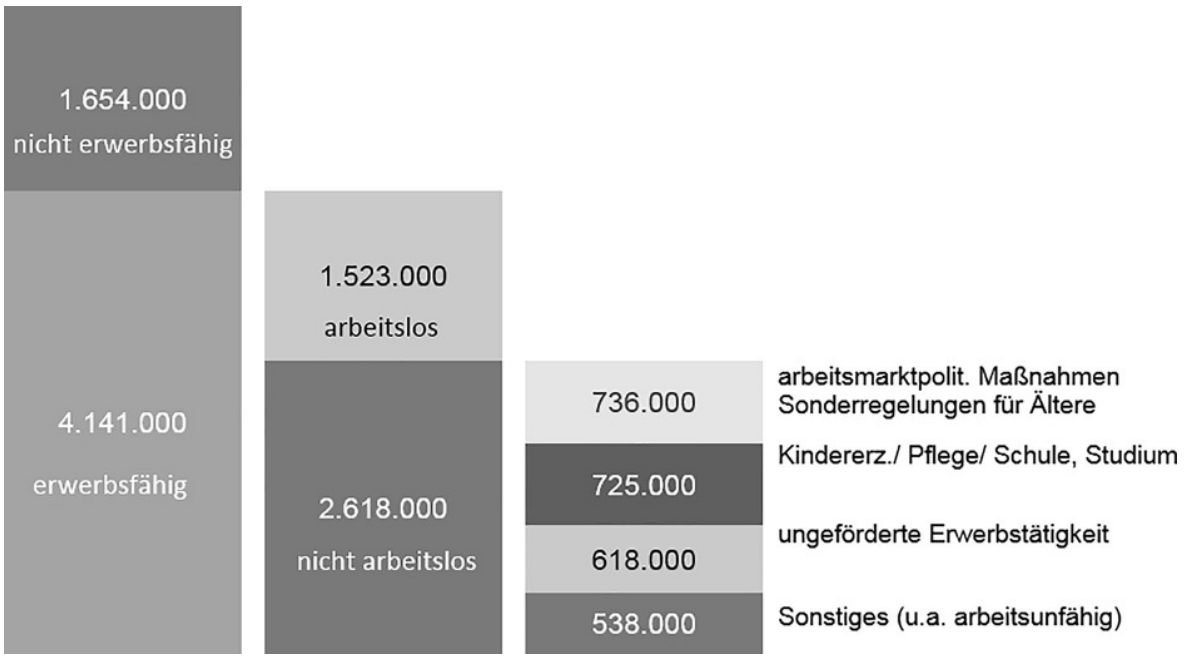

Abb. 3 Hilfebedürftigkeit und Arbeitslosigkeit, Deutschland, 2018

denen Arbeit nach $\S 10$ SGB II nicht zumutbar ist, werden wegen mangelnder Verfügbarkeit nicht als arbeitslos gezählt. Darunter fallen insbesondere Leistungsberechtigte, die Kinder erziehen, Angehörige pflegen oder zur Schule gehen. Abb. 3 zeigt die Größenordnungen der einzelnen Status.

Im Jahr 2018 waren mehr als drei Fünftel der erwerbsfähigen Leistungsberechtigten Arbeitslosengeld II-berechtigt ohne $\operatorname{arbeitslos}^{3} \mathrm{zu}$ sein. Gut jeder vierte nicht arbeitslose erwerbsfähige Leistungsberechtigte ging in die Schule, befand sich im Studium oder kümmerte sich um kleine Kinder bzw. pflegte Angehörige. Mehr als ein Viertel ging einer ungeförderten Erwerbstätigkeit von mindestens 15 Wochenstunden nach. Rund ein Fünftel befand sich in einer arbeitsmarktpolitischen Maßnahme.

Eine weitere Annahme über Personen in der Grundsicherung lautet: Bei Arbeitslosengeld II-beziehenden Menschen handele es sich um Personen im Langzeitleistungsbezug. Richtig ist: Es gibt Schnittmengen. Als langzeitleistungsbeziehende Person wird gezählt, wer erwerbsfähig ist, das 17. Lebensjahr vollendet hat und eine bisherige Verweildauer in der Grundsicherung von mindestens 21 Monaten in den letzten 24 Monaten erreicht. ${ }^{4}$ Derzeit sind mehr als zwei Drittel der erwerbsfähigen Leistungsberechtigten im Bestand als Langzeitleistungsbezieher einzuordnen. Darunter sind etwas weniger als zwei Fünftel arbeitslos.

\footnotetext{
3 Die Angaben zu den arbeitslosen erwerbsfähigen Leistungsberechtigten weichen von den Arbeitslosen im Rechtskreis SGB II ab, da wegen nachträglichen Änderungen im Leistungsstatus und kurzzeitigen Leistungsunterbrechungen (ohne Rechtskreiswechsel) nicht alle in der Arbeitslosenstatistik zum Stichtag erfassten SGB II-Arbeitslosen auch Leistungsempfänger in der Grundsicherung sind.

4 Zeiten der Nichterwerbsfähigkeit vor Vollendung des 15. Lebensjahres gehen nicht in die Dauerberechnung ein, weshalb der Langzeitleistungsbezug nur für Personen ab Vollendung des 17. Lebensjahres ermittelt wird.
} 
Diese beiden Beispiele zeigen, welche Kenntnisse der fachlichen Hintergründe erforderlich sind, um Statistiken zur Arbeitslosigkeit und Grundsicherung richtig zu lesen und richtig verstehen zu können. Sind diese Informationen nicht vorhanden, wird zwangsläufig über unbekannte Sachverhalte gesprochen, ohne es zu merken. Im Folgenden werden Maßnahmen bzw. Angebote beschrieben, die den Nutzern von BA-Statistiken das Lesen und Verstehen erleichtern sollen.

\section{Wie trägt die Statistik der BA zur Verbesserung der Statistical Literacy bei?}

Katherine Wallman beschreibt Statistical Literacy als die „Fähigkeit, statistische Ergebnisse, die unser tägliches Leben durchdringen, zu verstehen und kritisch zu bewerten - verbunden mit der Anerkennung des Beitrags von statistischem Denken zu öffentlichen wie privaten, beruflichen wie persönlichen Entscheidungen“ (Wallman 1993, S. 1). Das Verständnis für Statistik kann von verschiedenen Personen und Institutionen für Adressaten mit unterschiedlich ausgeprägten Vorkenntnissen auf vielfältige Art und Weise gefördert werden. Die Ergebnisse fallen dabei umso besser aus, je mehr die Angebote ineinandergreifen und aufeinander aufbauen. Die Amtliche Statistik ist hier neben beispielsweise den Bildungseinrichtungen oder den Statistischen Gesellschaften ein wichtiger Akteur bei der Vermittlung von statistischen Kenntnissen. Sie kann oder sollte über die von ihr erhobenen Daten und deren Aussagekraft, die verwendeten Methoden sowie typische Fallstricke und Missverständnisse informieren (vgl. Ferligoj 2015 und UNECE 2014). Dies kann entweder direkt oder aber auch indirekt über die Medien geschehen.

Als mögliche direkte Ansätze der Amtlichen Statistik, in der Öffentlichkeit auf ein verbessertes Verständnis von Statistik und Statistiken hinzuwirken, nennt Ferligoj:

1. die Bereitstellung von kurzen und leicht verständlichen Informationen,

2. das Angebot von themen- und kundenspezifischen Seminaren und Vorträgen,

3. die Unterstützung der Nutzer der Statistiken bei der Verwendung und Interpretation der Daten und

4. die Darstellung von typischen Missverständnissen und Fehlinterpretationen.

Die Statistik der BA ist Teil des Systems der amtlichen Statistik in Deutschland und in Europa. Neben mehreren zentralen Funktionsbereichen in Nürnberg stehen als weitere Ansprechpartner bundesweit fünf regionale Statistik-Services zur Verfügung. Während der zentrale Statistik-Service und die Arbeitsmarktberichterstattung in Nürnberg vor allem die Entwicklungen auf Bundesebene als Aufgabe im Blick haben, stehen in den Statistik-Services jeweils die regionalen Arbeitsmärkte auf Landes-, Agentur-, Kreis- und Gemeindeebene und der Kontakt zu den regionalen Arbeitsmarktakteuren im Mittelpunkt.

Als Teil des Systems der amtlichen Statistik steht die Statistik der BA in der Verantwortung, ihren Teil zur Verbesserung des statistischen Verständnisses beizutragen. Wie und inwieweit sie die genannten Ansätze verfolgt, soll im Folgenden dargestellt werden. 


\subsection{Bereitstellung von kurzen und leicht verständlichen Informationen}

Die Statistik der BA veröffentlicht regelmäßig Daten zu verschiedenen Aspekten des Arbeits- und Ausbildungsmarkts. Die schiere Fülle an Daten bedingt häufig die Bereitstellung der Daten in Form von Tabellenheften. Viele Nutzer können mit so publizierten Daten gut umgehen und arbeiten. Fehlt es aber an statistischen Vorkenntnissen oder an Hintergrundwissen zu beispielsweise gesetzlichen Regelungen, die die Daten beeinflussen, kann es zu Fehlinterpretationen kommen. Häufig fehlt den Nutzern auch schlicht die Zeit, sich aus der Fülle an Daten alle für sie relevanten Informationen herauszufiltern. Eine Reduzierung von Umfang und Komplexität der verfügbaren Daten verbunden mit einer anderen Darstellungsform hilft daher, einen breiteren Adressatenkreis mit dem statistischen Angebot zu erreichen. Um bei den Planungs- und Entscheidungsprozessen von lokalen, regionalen und überregionalen Akteuren und Netzwerken möglichst viele potentielle Interessenten mit umfassenden und verständlichen Informationen zu unterstützen, enthält die Produktlandschaft der Statistik der BA daher ergänzend zu den Tabellenheften weitere Angebote.

Vielen Menschen fällt es leichter, Informationen aufzunehmen, wenn diese visuell aufbereitet sind. Dieser Tatsache tragen die Visualisierungen Rechnung, die im Internetangebot der Statistik der BA (https://statistik.arbeitsagentur.de/) zu verschiedenen Themen angeboten werden. Nutzer, die sich einen Überblick über bestimmte Sachverhalte verschaffen wollen, erhalten dort beispielsweise in „Berufe auf einen Blick" graphisch aufbereitet die zentralen Informationen wie Beschäftigung, Arbeitslosigkeit und Fachkräftebedarf zu einem sie interessierenden Beruf oder können mit Hilfe der "Arbeitsmarktanalyse“ regional differenziert die Ausgleichsprozesse am Arbeitsmarkt anhand von Abbildungen detailliert betrachten. ${ }^{5}$

Broschüren und Kompaktinformationen setzen ebenfalls auf graphisch aufbereitete statistische Arbeitsmarktinformationen, enthalten aber zusätzlich ausformulierte Erläuterungen zur dargestellten Thematik. So sind statistische Arbeitsmarktdaten häufig von Änderungen der rechtlichen Rahmenbedingungen betroffen; werden diese Änderungen übersehen, können Fehlinterpretationen die Folge sein, weil vermeintliche arbeitsmarktliche Veränderungen in Wahrheit beispielweise einer veränderten Gesetzeslage zuzuschreiben sind. Exemplarisch seien die Arbeitslosigkeit und die Beschäftigung von älteren Menschen genannt, deren Entwicklung nicht nur von demographischen Faktoren, sondern auch von Änderungen des Renteneintrittsalters abhängig ist. Einen ähnlichen Adressatenkreis hat die Reihe Statistik erklärt im Blick. Hier werden häufig gestellte Fragen kurz und bündig beantwortet, so dass auch ohne vertiefte Fachkenntnisse Inhalte nachvollziehbar sind. Diese Fragen lauten zum Beispiel: „Was ist der Unterschied zwischen Arbeitslosigkeit und Erwerbslosigkeit?“, „Wie wird eigentlich die Arbeitslosenquote berechnet?“ oder „Wieso gibt es eigentlich Arbeitslosengeldempfänger, die nicht arbeitslos sind?“.

Wirkung entfalten Maßnahmen zur Förderung des sicheren Umgangs mit Statistik und Statistiken am besten, wenn die Angebote der einzelnen Akteure ineinandergreifen. Als Beitrag zur Unterstützung der Bildungseinrichtungen stellt die Statistik der

\footnotetext{
5 Die Visualisierungen der Statistik der BA finden sich unter https://statistik.arbeitsagentur.de/Navigation/ Statistik/Statistische-Analysen/Interaktive-Visualisierung/Interaktive-Visualisierung-Nav.html.
} 
BA daher Lernmaterialien zur Verfügung, die kompakt aufbereitet zentrale Aspekte der Arbeitsmarktstatistik erläutern und zur Diskussion einladen. Ziel ist es, Schüler und Studenten dazu zu ermuntern, sich mit den Möglichkeiten und Grenzen von Statistik auseinanderzusetzen, sich anhand von Fakten selbst eine Meinung zu bilden und zu lernen, sich kritisch mit Daten, Informationen oder auch Schlagzeilen auseinanderzusetzen.

Das Angebot speziell für Studenten beschränkt sich nicht auf Lehrmaterialien, sondern umfasst auch Veranstaltungen an und direkte Kooperationen mit Universitäten. Mitarbeiter der Statistik der BA bieten an einigen Hochschulen Veranstaltungen an, um Studierenden über die theoretische Wissensvermittlung hinaus Kenntnisse im praktischen Umgang mit Statistiken - speziell Arbeitsmarktstatistik - zu vermitteln. Als Projektpartner von Hochschulen engagiert sich die Statistik der BA zudem als Themengeber und mit Anwendungsfällen bei der Erstellung von Praxisarbeiten. Zudem ist sie Teil von EMOS (European Master in Official Statistics; https://ec.europa. eu/eurostat/web/european-statistical-system/emos), einem gemeinsamen Projekt von Hochschulen und amtlicher Statistik mit dem Ziel, die Zusammenarbeit zwischen Hochschulen und Produzenten amtlicher Statistiken zu stärken und Fachleute heranzubilden, die mit den europäischen amtlichen Daten auf verschiedenen Ebenen umzugehen wissen.

\subsection{Themen- und kundenspezifische Seminare und Vorträge}

Eine weitere Möglichkeit, Hintergründe und Zusammenhänge der Statistiken zum Arbeits- und Ausbildungsmarkt zugänglich zu machen, sind Vorträge zu speziellen Arbeitsmarktthemen. Der Adressatenkreis der Statistik der BA umfasst hier sowohl Kollegen innerhalb der Bundesagentur für Arbeit als auch Externe, beispielsweise bei Vorträgen im Rahmen von Fachveranstaltungen, auf denen Vortragende der Statistik der BA Impulsvorträge beisteuern.

Daneben führt die Statistik/Arbeitsmarktberichterstattung der BA alle zwei Jahre eine Informationsveranstaltung für die akademischen Berater der Arbeitsagenturen durch, auf der unter anderem auch statistische Informationen zum Arbeitsmarkt speziell für Akademiker präsentiert und erläutert werden. Da die Teilnehmer an der Veranstaltung als Multiplikatoren für ihre Kollegen, aber auch nach außen wirken, können Zusammenhänge, Hintergründe und Erläuterungen so einem breiten Adressatenkreis zugänglich gemacht werden.

Auf den Multiplikatoreffekt setzt auch das Engagement der Statistik der BA beim Presseseminar der Bundesagentur für Arbeit. Diese jährlich durchgeführte Veranstaltung bietet insbesondere Journalisten, zu deren Aufgabengebiet die Berichterstattung über den Arbeitsmarkt gehört, die Möglichkeit, sich im direkten Austausch mit der Bundesagentur für Arbeit über neue Entwicklungen und Projekte zu informieren. Da Vertreter von Presse und Medien mit ihrer Arbeit ein breit gefächertes Publikum erreichen, sind sie wichtige Multiplikatoren für die Verbreitung statistischer Kenntnisse. Nach Bedarf sind Mitarbeiter der Statistik der BA daher bei dieser Veranstaltung mit einem Informationsstand, einem Vortrag oder einem Workshop vertreten und informieren über Hintergründe der Arbeitsmarktstatistiken, beispielsweise die oben beschriebenen Zusammenhänge zwischen Arbeitslosigkeit und Unterbeschäftigung. 
Für Fach- und Führungskräfte der BA oder der Jobcenter, die Kenntnisse zu speziellen Statistiken erwerben oder vertiefen wollen, werden von der Statistik der BA im Bildungskatalog der Bundesagentur für Arbeit Schulungen zu ausgewählten Statistiken und Themen angeboten. Interessenten können auf diesem Weg in einzelnen Schulungsmodulen den korrekten Umgang mit der Beschäftigtenstatistik, der Ausbildungsmarktstatistik, der Grundsicherungsstatistik und der Förderstatistik erlernen; sie können die typischen Interpretationsfehler und Verwechslungen kennenlernen; oder sie erfahren, welche statistischen Informationen zu einer Marktanalyse oder einer Beschreibung des Fachkräftebedarfs sinnvoll herangezogen werden können. Für regionale Arbeitsmarktakteure führen die regionalen Statistik-Services themenund kundenspezifische Schulungen durch. Hier geht es um das Erlernen von Grundlagen der Statistiken und um die Vertiefung von Kenntnissen in der praktischen Anwendung, verbunden mit den Fragen, was die Daten bedeuten und was sie aussagen. Auch Fachvorträge bei verschiedenen internen und externen Adressatenkreisen werden angefragt und gehalten.

\subsection{Unterstützung bei der Interpretation und Verwendung von Daten}

Im Selbstverständnis der Statistik/Arbeitsmarktberichterstattung der Statistik der BA (vgl. Bundesagentur für Arbeit 2019b) wird unter anderem auf Service-Orientierung sowie fachkundige und unterstützende Beratung abgestellt. Dazu gehört auch die Bereitstellung von aufbereiteten, unter Umständen mit Erläuterungen versehenen, statistischen Informationen und Analysen. Dies geschieht häufig in Form von Präsentationsfolien, ergänzt um Notizen mit Hinweisen zu Aussagekraft, Interpretation und Einschränkung der dargestellten Daten. Diese Mustervorträge - insbesondere zum Arbeitsmarkt für Akademiker und über die regionale Arbeitsmarktentwicklung - können komplett oder in Auszügen von Kollegen innerhalb und außerhalb der Statistik der BA als Grundlage für eigene Veranstaltungen und Vorträge verwendet werden. Derartige Materialien sind auch Grundlage von internen Informationsveranstaltungen für Kollegen. Anders als die im Bildungskatalog angebotenen Schulungsmodule werden sie adressaten- und anlassbezogen konzipiert und in größeren zeitlichen Abständen bei entsprechendem Interesse durchgeführt.

Auch für Datennutzer, die über gute oder sehr gute Kenntnisse statistischer Methoden verfügen, ist Unterstützung beim Umgang mit Daten wichtig, da das Wissen um den Kontext der Daten für deren Analyse und Bewertung wesentlich ist (Gal 2002). Hierzu gehören gut dokumentierte Hintergrundinformationen zu den Datenquellen oder den Erhebungsmethoden. Im Einklang mit dem Verhaltenskodex für europäische Statistiken (2017) macht die Statistik der BA neben den Statistiken die Metadaten und Erläuterungen entsprechend dem Grundsatz der Unparteilichkeit verfügbar und zugänglich. Datenherkunft, Methoden, Definitionen und Informationen zur Qualität von Ergebnissen einzelner Statistiken sowie Verwendungsmöglichkeiten - Grundlage einer korrekten Interpretation statistischer Ergebnisse - werden in Qualitätsberichten transparent gemacht.

Neue Daten und Erweiterungen von Statistiken werden mit Methodenberichten eingeführt, um die Genese und Aussagekraft vor der ersten Veröffentlichung transparent zu machen. Für den schnellen und weniger detaillierten Blick auf die rele- 
vanten Begriffe und verwendeten Kennzahlen aus den Produkten der Statistik der BA sind zudem ein Glossar und eine Reihe von Kennzahlensteckbriefen abrufbar; letztere beschreiben die Zusammensetzung von Zähler und Nenner für verschiedene Kennzahlen und geben Hinweise zum Verwendungszweck und zur Aussagekraft. Beispiele dafür sind das Entlassungsrisiko oder die Zugangsrate Arbeitsloser. Für Nutzer, die eher an konkreten Daten als an den methodischen Hintergründen interessiert sind, begleitet die Statistik der BA die Einführung neuer Produkte seit kurzem mit kurzen Filmen ${ }^{6}$, in denen die neuen Daten und ihre Aussagekraft leicht verständlich erklärt werden.

Neben diesem proaktiven Angebot an Interpretationshilfen erfolgt ein großer Teil der Unterstützung bei der Verwendung und Interpretation von statistischen Arbeitsmarktinformationen reaktiv in Form von Antworten auf Einzelanfragen. Unterschiedlichste Kunden - z.B. Vertreter von Politik, Wirtschaft, Medien und Forschung, regionale Arbeitsmarktakteure, insbesondere Mitarbeiter von Arbeitsagenturen und Jobcentern, aber auch interessierte Einzelpersonen - wenden sich mit konkreten Datenanfragen oder auch lediglich grob umrissenen Problemstellungen an die Statistik der BA. Im individuellen Kundendialog werden hier Anfragen operationalisiert, Aussagekraft der Daten und Zusammenhänge erläutert, ggf. alternative Lösungen gesucht und zuweilen auch Irrtümer ausgeräumt. Je nach Kunde bzw. Kundengruppe werden dabei die jeweiligen Vorkenntnisse und auch spezifischen Probleme berücksichtigt. Die Unterstützung speziell von regionalen Arbeitsmarktakteuren beinhaltet neben Hinweisen zur richtigen Verwendung und Interpretation von Daten beispielsweise oft auch Beratung zur Problematik von (zu) kleinen Fallzahlen und daraus resultierenden Einschränkungen.

\subsection{Darstellung typischer Fehlinterpretationen und Missverständnisse}

Die Darstellung und Korrektur von typischen Fehlinterpretationen und Missverständnissen ist streng genommen kein eigener Handlungsstrang in den Aktivitäten zur Verbesserung der statistischen Kompetenz. Vielmehr zieht sie sich als roter Faden durch viele der zuvor beschriebenen Aktivitäten der Statistik der BA. Um es gar nicht erst zu Fehlinterpretationen bei den Anwendern und Nutzern der Daten kommen zu lassen, sind die methodischen Hintergründe und Informationen zu Datenherkunft und Aussagekraft in den Qualitäts- und Methodenberichten dokumentiert. Über bereits in vielen Köpfen steckende Irrtümer - zum Beispiel die beiden oben diskutierten Fehleinschätzungen, die Arbeitslosenzahl würde künstlich kleingerechnet und alle Bezieher von Hartz IV seien langzeitarbeitslos - wird in Vorträgen, Lernund Schulungsunterlagen, in Broschüren und vor allem im Kontakt mit potenziellen Multiplikatoren beharrlich aufgeklärt. Allerdings lassen sich Fehlinterpretationen statistischer Daten und Missverständnisse nicht völlig vermeiden. Sollte es dennoch zu Missverständnissen kommen, beispielsweise durch Pressevertreter, die bei der Fülle ihrer Themen nicht überall über Detailkenntnisse verfügen können, steht die Statistik der BA in engem Austausch mit der Pressestelle der Bundesagentur für

\footnotetext{
6 Zum Beispiel: https://statistik.arbeitsagentur.de/Navigation/Statistik/Statistische-Analysen/InteraktiveVisualisierung/Strukturwandel-nach-Berufen/Strukturwandel-nach-Berufen-Nav.html.
} 
Arbeit. Als Multiplikator und Ansprechpartner für Journalisten kann diese im direkten Austausch bereits entstandene fehlerhafte Interpretationen korrigieren bzw. versuchen eine Wiederholung zu vermeiden.

\section{Zusammenfassung}

Ein grundlegendes statistisches Verständnis ist wichtig, um mit der wachsenden Menge an verfügbaren Daten, die unseren Alltag umgeben, umgehen zu können. Statistische Kompetenz dreht sich mehr um Fragen als um Antworten und sollte dabei helfen, bessere Fragen zu stellen und damit bessere Urteile zu fällen und bessere Entscheidungen zu treffen (vgl. Schield 1999). Generell ergeben sich in der inhaltlichen Auseinandersetzung mit Daten idealerweise folgende Fragen: Was wird dargestellt oder abgebildet? Woher stammen die Daten? Kann man dieser Datenquelle vertrauen? Sind die Rückschlüsse, die man aus den Daten zieht, vertretbar? Oder sind die Folgerungen zu unsicher?

Die verfügbare und zugängliche Datenmenge steigt in den meisten Themenbereichen deutlich an. Der Arbeitsmarkt gehört dabei zu den Themenfeldern, die für viele Menschen wichtig und in ihrem Alltag präsent sind: sie sind arbeitslos, beschäftigt oder auf finanzielle Unterstützung angewiesen; sie informieren sich in der Zeitung, dem Internet, sehen Fernsehnachrichten; sie sind beteiligt an politischen Entscheidungen den Arbeitsmarkt betreffend; sie haben als Arbeitgeber Probleme, ihre offenen Stellen zu besetzen. Um alle adressatengerecht bestmöglich zu unterstützen, arbeitet die Statistik der BA an der Verbesserung des statistischen Verständnisses. Sie hält die Richtlinien der amtlichen Statistik und des Europäischen Statistischen Systems ein - auch um deutlich zu machen, dass ihren Datenquellen vertraut werden kann.

Open Access Dieser Artikel wird unter der Creative Commons Namensnennung 4.0 International Lizenz (http://creativecommons.org/licenses/by/4.0/deed.de) veröffentlicht, welche die Nutzung, Vervielfältigung, Bearbeitung, Verbreitung und Wiedergabe in jeglichem Medium und Format erlaubt, sofern Sie den/die ursprünglichen Autor(en) und die Quelle ordnungsgemäß nennen, einen Link zur Creative Commons Lizenz beifügen und angeben, ob Änderungen vorgenommen wurden.

\section{Literatur}

Bundesagentur für Arbeit (2009) Umfassende Arbeitsmarktstatistik: Arbeitslosigkeit und Unterbeschäftigung. Grundlagen: Methodenbericht. https://statistik.arbeitsagentur.de/Statischer-Content/ Grundlagen/Methodik-Qualitaet/Methodenberichte/Arbeitsmarktstatistik/Generische-Publikationen/ Methodenbericht-Arbeitslosigkeit-Unterbeschaeftigung.pdf. Zugegriffen: 09. Juli 2019

Bundesagentur für Arbeit (2013) Vervollständigung der Datenbasis für die Unterbeschäftigung. Grundlagen: Methodenbericht. https://statistik.arbeitsagentur.de/Statischer-Content/Grundlagen/MethodikQualitaet/Methodenberichte/Arbeitsmarktstatistik/Generische-Publikationen/Methodenbericht-Unter beschaeftigung-integriert.pdf. Zugegriffen: 09. Juli 2019

Bundesagentur für Arbeit (2015) Warum sind nicht alle erwerbsfähigen Leistungsbezieher arbeitslos? Grundlagen: Methodenbericht. https://statistik.arbeitsagentur.de/Statischer-Content/Grundlagen/ Methodik-Qualitaet/Methodenberichte/Grundsicherung-Arbeitsuchende-SGBII/Generische-Publika tionen/Methodenbericht-Warum-sind-nicht-alle-eLb-arbeitslos.pdf. Zugegriffen: 09. Juli 2019

Bundesagentur für Arbeit (2018) Statistik der Grundsicherung für Arbeitsuchende nach dem SGB II. Grundlagen: Qualitätsbericht. https://statistik.arbeitsagentur.de/Statischer-Content/Grundlagen/ 
Methodik-Qualitaet/Qualitaetsberichte/Generische-Publikationen/Qualitaetsbericht-Statistik-Grund sicherung-Arbeitsuchende-SGBII.pdf. Zugegriffen: 09. Juli 2019

Bundesagentur für Arbeit (2019a) Statistik der Arbeitslosen, Arbeitsuchenden und gemeldeten erwerbsfähigen Personen. Grundlagen: Qualitätsbericht. https://statistik.arbeitsagentur.de/Statischer-Content/ Grundlagen/Methodik-Qualitaet/Qualitaetsberichte/Generische-Publikationen/QualitaetsberichtStatistik-Arbeitslose-Arbeitsuchende.pdf. Zugegriffen: 09. Juli 2019

Bundesagentur für Arbeit (2019b) Arbeits- und Entwicklungsprogramm der Statistik der BA 2019-2020. https://statistik.arbeitsagentur.de/Statischer-Content/Service/Ueber-uns/Arbeits-und-Entwicklungspro gramm/Generische-Publikationen/Arbeits-Entwicklungsprogramm-2019-2020.pdf. Zugegriffen: 09. Juli 2019

Ferligoj A (2015) How to improve statistical literacy? Metodol Zvezki 12(1):1-10

Gal I (2002) Adults' statistical literacy: meaning, components, responsibilities. Int Stat Rev 70:1-51

Schield M (1999) Statistical literacy: thinking critically about statistics. Significance 1:15-20

UNECE (2014) Making Data Meaningful. Part 4: How to improve statistical literacy: A guide for statistical organizations. United Nations, New York, Geneva

Verhaltenskodex für europäische Statistiken (2017) https://ec.europa.eu/eurostat/documents/4031688/ 9394019/KS-02-18-142-DE-N.pdf. Zugegriffen: 09. Juli 2019

Wallman KK (1993) Enhancing statistical literacy: enriching our society. J Am Stat Assoc 88(421):1-8

Hinweis des Verlags Der Verlag bleibt in Hinblick auf geografische Zuordnungen und Gebietsbezeichnungen in veröffentlichten Karten und Institutsadressen neutral. 